\title{
3D-Printed Drug Delivery Systems: The Effects of Drug Incorporation Methods on Their Release and Antibacterial Efficiency
}

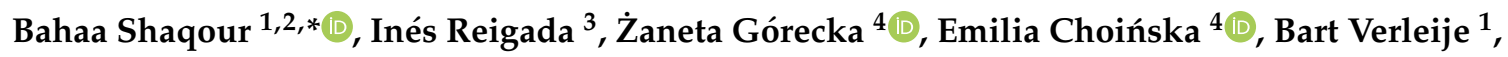 \\ Koen Beyers ${ }^{1}$, Wojciech Święszkowski ${ }^{4}$, Adyary Fallarero ${ }^{3}$ and Paul $\operatorname{Cos}^{2} \mathbb{C}$ \\ 1 Voxdale bv, Bijkhoevelaan 32C, 2110 Wijnegem, Belgium; bart@voxdale.be (B.V.); koen@voxdale.be (K.B.) \\ 2 Laboratory for Microbiology, Parasitology and Hygiene (LMPH), Faculty of Pharmaceutical, Biomedical and \\ Veterinary Sciences, University of Antwerp, Universiteitsplein 1 S.7, 2610 Antwerp, Belgium; \\ paul.cos@uantwerpen.be \\ 3 Drug Research Program, Division of Pharmaceutical Biosciences, Faculty of Pharmacy, University of \\ Helsinki, 00790 Helsinki, Finland; ines.reigada@helsinki.fi (I.R.); adyary.fallarero@helsinki.fi (A.F.) \\ 4 Faculty of Materials Sciences and Engineering, Warsaw University of Technology, Woloska 141, \\ 02-507 Warsaw, Poland; gorecka.zaneta@gmail.com (Ż.G.); choinska.emilia@gmail.com (E.C.); \\ wojciech.swieszkowski@pw.edu.pl (W.Ś.) \\ * Correspondence: bahaa@voxdale.be
}

Received: 3 July 2020; Accepted: 27 July 2020; Published: 29 July 2020

\begin{abstract}
Additive manufacturing technologies have been widely used in the medical field. More specifically, fused filament fabrication (FFF) 3D-printing technology has been thoroughly investigated to produce drug delivery systems. Recently, few researchers have explored the possibility of directly 3D printing such systems without the need for producing a filament which is usually the feedstock material for the printer. This was possible via direct feeding of a mixture consisting of the carrier polymer and the required drug. However, as this direct feeding approach shows limited homogenizing abilities, it is vital to investigate the effect of the pre-mixing step on the quality of the 3D printed products. Our study investigates the two commonly used mixing approaches-solvent casting and powder mixing. For this purpose, polycaprolactone $(\mathrm{PCL})$ was used as the main polymer under investigation and gentamicin sulfate (GS) was selected as a reference. The produced systems' efficacy was investigated for bacterial and biofilm prevention. Our data show that the solvent casting approach offers improved drug distribution within the polymeric matrix, as was observed from micro-computed topography and scanning electron microscopy visualization. Moreover, this approach shows a higher drug release rate and thus improved antibacterial efficacy. However, there were no differences among the tested approaches in terms of thermal and mechanical properties.
\end{abstract}

Keywords: fused filament fabrication; 3D printing; drug loading; drug release

\section{Introduction}

Additive manufacturing (AM), also known as 3D printing, has been extensively explored in the past decade. This technology has helped to pave the way for personalized and customized production. Fused deposition modeling $\left(\mathrm{FDM}^{\mathrm{TM}}\right)$, also known as fused filament fabrication (FFF), is one of the 3D printing technologies that was widely used after the expiration of the Stratasys patent in 2009 [1]. As this technology is cheaper and more flexible than other 3D printing technologies, many studies explored its potential in fields such as personalized medicine [2]. The focus was on producing patient specific drug delivery systems, such as tablets for oral drug delivery or implants for localized drug delivery. 
The typical approach for 3D printing a drug delivery system includes: (1) selection of a carrier polymer and a required active pharmaceutical ingredient (API); (2) preparing a filament using the hot melt extrusion (HME) process; (3) designing the geometry of the drug loaded device; and 4) 3D printing [3].

The step that involves the filament preparation can be omitted if a 3D printer was designed to be compatible with the polymer and the drug in their original form as a raw material. Interestingly, only a few studies have used such systems [4-6], while the vast majority of studies have used the previously mentioned approach. There are many reasons for the limited interest in systems that do not include the filament preparation step in the process. Among them is the well-established knowledge of HME technologies. Another reason is the complex optimization process and parameter setting in these 3D printers. This complexity is due to the extrusion system used in these printers, which can either be a screw-based or pneumatic extrusion system or both. Moreover, with this configuration a challenge arises, which is to achieve a homogenous mixture between the carrier polymer and the API, especially when the latter is much lower in volume or there is a large difference in size, such as pellets, chips, or powders. As there are countless approaches for pre-mixing the polymer and the drug, it was noticed that a standardized method for the loading of an API into a carrier polymer for 3D printing purposes is not available [7].

In this study, gentamicin sulfate (GS) was selected as a model drug. It is an aminoglycoside antibiotic used to treat several types of bone and soft tissue infections [8]. It has a high solubility in water [9]; however, its effectiveness is concentration-dependent, especially for the treatment of biofilms. These kinds of infections are often hospital-acquired and associated with medical device use, being up to $80 \%$ of these infections caused by bacterial biofilms [10]. Biofilms are defined as a community of cells encased within a self-produced matrix that adheres to biological or non-biological surfaces [11,12]. By switching into the biofilm state, bacteria can withstand antibiotic chemotherapy, increasing the minimum inhibitory concentration (MIC) up to 1000 times [13], which is increasingly regarded as the most important nonspecific mechanism of antimicrobial resistance $[14,15]$. This is especially true for orthopedic implants, where biofilm-associated infections cause up to $25 \%$ of failures [16].

The implantation of a foreign material represents per se a major risk factor for the development and chronic progression of osteomyelitis. Presently, the commonly used method of prevention is systemic administration of oral antibiotics, but this can result in adverse effects and the generation of bacterial resistance. Drug delivery systems have many advantages, among them is the delivery of API at the targeted physical site for a prolonged period and at the required therapeutic level [17]. Additionally, 3D printing can be used to produce drug eluting medical devices that may provide the proper concentration of API.

Moreover, the use of GS in this approach has proven efficacy because the system based on GS loaded into Poly(methyl methacrylate) (PMMA) beads is widely used for infection eradication in orthopedic applications. As carrier polymer to be investigated, polycaprolactone (PCL) was selected. This has been extensively explored for applications such as tissue engineering and drug-delivery systems due to its biocompatibility and biodegradability $[18,19]$. Loading PCL with antibiotics such as GS has shown great potential during recent years. Such drug delivery systems have been extensively explored among the literature $[20,21]$. It was shown that the produced systems can prevent bacterial infections and biofilm formation both in vitro and in vivo.

This study compares two mixing approaches widely used in the literature. The first approach is powder mixing. In this approach the carrier polymer and the drug are simply mixed physically; however, it is very important that they have similar forms in terms of geometry to assure homogenous mixing before 3D printing. Saviano et al. [22] discussed the effect of polymer size on the homogeneity of the drug distribution in drug loaded devices and also the extrudability of the mixture. They tested a range of polymer sizes from powders with less than $250 \mu \mathrm{m}$ particle size up to pellets with diameters of 4 to $5 \mathrm{~mm}$. They concluded that the moderate size particles, ranging from 250 to $600 \mu \mathrm{m}$, allow for homogeneous drug dispersion in the polymer matrix and good processability. On the other hand, a 
similar effect can be obtained by the second mixing method, i.e., the solvent casting technique. In this approach, an organic solvent is used to dissolve the polymer and then the drug is suspended in the system [23]. Subsequently, the solution/suspension is cast, and the solvent can evaporate. However, it includes the use of relatively large volumes of solvents, which can be toxic, and make the process not environmentally friendly.

The aim of this study was to shed light on the preparation methods of drug loaded printable materials. This very important issue has not been thoroughly discussed in the literature. As the production of drug loaded devices using 3D printing technologies has been intensively investigated over recent years, the methods of material preparation or drug incorporation have varied between the different studies. Additionally, there are no standardized methods of drug incorporation into a polymeric carrier prior to 3D printing, which makes it difficult to compare between published results. Moreover, with the new trend of single step 3D printing, it is very important to investigate the most effective drug loading approaches for this process. Thus, evaluation of material preparation techniques and the effects of different incorporation methods on drug distribution, release, and antibacterial efficiency is key for producing standard drug loading and incorporation techniques.

\section{Materials and Methods}

PCL pellets (Mn 80,000, cat no.: 440744-500G) were obtained from Sigma Aldrich (Gillingham, U.K.), GS, USP grade was obtained from Fluorochem Ltd. (Glossop, U.K.). Solvents used such as dichloromethane (DCM), chloroform, ethanol, and iso-propanol, and components to prepare the phthaldialdehyde reagent were obtained from Chempur (Piekary Śląskie, Poland). Tryptic Soy Broth (TSB) and tryptic soy agar (TSA) were obtained from Neogen ${ }^{\circledR}$ (Lansing, MI, USA).

\subsection{Preparation of Mixtures}

PCL was loaded with GS at two concentrations $2.5 \%$ and $7.5 \%(w / w)$. In the solvent casting approach, the mixture was prepared by suspending the proper amount of drug (based on the required drug loading) in $100 \mathrm{~mL}$ of DCM using a magnetic stirrer (VELP Scientifica Srl, Usmate, Italy) for $10 \mathrm{~min}$. Subsequently, $10 \mathrm{~g}$ of PCL was added and stirred overnight to form a $10 \%(w / v)$ solution. The polymer solution with suspended drug was then poured on a petri dish $(\phi 200 \mathrm{~mm})$ to evaporate the solvent under the fume hood for two days. Finally, the films were vacuum dried for three days at $25^{\circ} \mathrm{C}$ and $50 \mathrm{mbar}$. Then, $10 \mathrm{~mm} \times 10 \mathrm{~mm}$ square pieces of drug loaded PCL were cut for printing.

In the powder mixing approach, the mixture was prepared by grinding PCL pellets using a grinder (Retsch ZM200, Katowice, Poland). First, pellets were frozen using liquid nitrogen, then grinded at $60,000 \mathrm{rpm}$ and sieved (aperture $0.75 \mathrm{~mm}$ ). The grinder was kept chilled by pouring small portions of liquid nitrogen during the grinding process. Subsequently, the polymer was added to $50 \mathrm{~mL}$ tubes. Then, the proper amount of drug was added, and the mixture was vortexed well for 5 min. Finally, the mixture was fed to a Bioscaffolder (SYSENG, Salzgitter, Germany). Figure 1A illustrates the two mixing approaches.

A

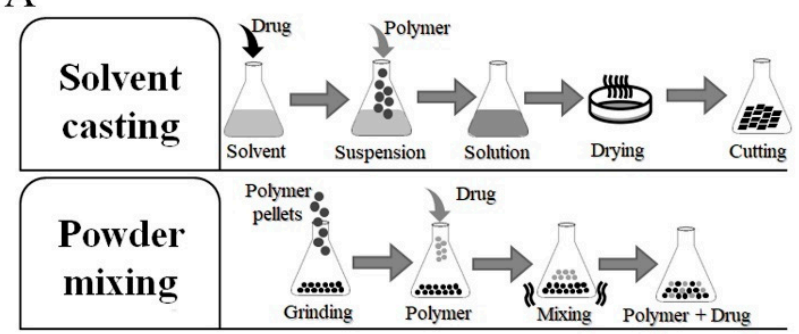

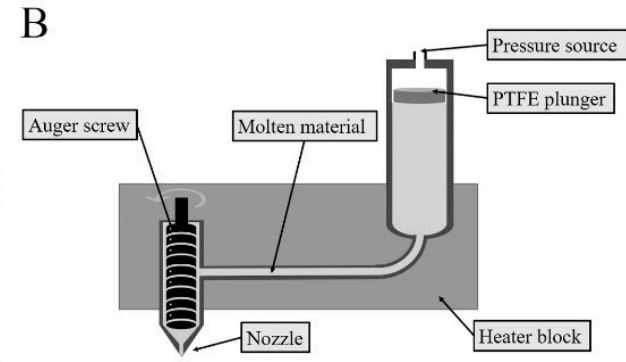

Figure 1. (A) Steps performed for preparing the drug-polymer mixture via solvent casting and powder mixing approaches and (B) The 3D printer extruder used for printing the samples. 


\subsection{Preparation of Samples}

The models of samples for printing were designed in SolidWorks (v.2017) software. Samples were 3D printed using a Bioscaffolder (SYSENG, Salzgitter, Germany) (printing head scheme presented in Figure 1B) and a dedicated Bioscaffolder-software (PrimCam v.3.0) for the slicing process. A nozzle with $1 \mathrm{~mm}$ diameter was used. The temperature of printing was set to $100^{\circ} \mathrm{C}$. The $\mathrm{X}$ and $\mathrm{Y}$ axes feed rate was set to $30 \mathrm{~mm} / \mathrm{min}$, the screw extruder rotation was set at $38 \mathrm{rpm}$ and a pneumatic pressure of 5 bar was applied. Three types of samples were prepared. For $\mu \mathrm{CT}$ scanning and tensile testing, single fibers were extruded through the nozzle by printing infill of rectangular single layer of cuboid with $2 \mathrm{~mm}$ distance between each line. A special platform (Figure 2) for the printing bed was prepared, which enabled printing of around $1 \mathrm{~mm}$ of the start and the end of the line on the platform, while the rest was hung in the air [24]. For release tests, disk-shaped samples with a diameter and height of $10 \mathrm{~mm}$ and $2 \mathrm{~mm}$ respectively, were designed and printed directly on a printing platform. The infill density was set to $100 \%$. The layer thickness was $0.8 \mathrm{~mm}$. Samples for the compression test were cut out from similarly printed bulk material with a biopsy puncher to achieve a repeatable cylinder with a $4 \mathrm{~mm}$ diameter. The height of these samples was also $4 \mathrm{~mm}$.

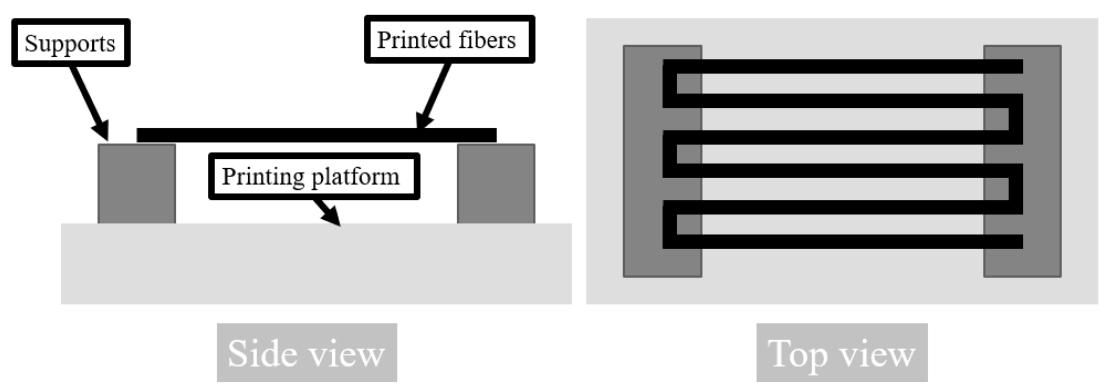

Figure 2. Setup for 3D printing of fibers.

\subsection{Scanning Electron Microscopy (SEM) Analysis}

The images of drug and polymer particles were acquired using a scanning electron microscope (SEM) (Phenom Pro X, Phenom-World, Eindhoven, The Netherlands) equipped with a holder dedicated for testing of non-conductive samples. The $10 \mathrm{kV}$ acceleration voltage was applied. Prior imagining samples were sputtered with a $7 \mathrm{~nm}$ conductive gold layer.

\subsection{Micro-Computed Tomography $(\mu \mathrm{CT})$}

$\mu \mathrm{CT}$ measurements were performed on the 2 mm-long fibers using SKYSCAN 1172 (Bruker, Kontich, Belgium). The scan was performed at $40 \mathrm{kV}$ and $250 \mathrm{~mA}$ over 180 degrees with a rotation step of $0.5^{\circ}$ and exposure time of $600 \mathrm{~ms}$. The image pixel size was $1.46 \mu \mathrm{m}$. The obtained planar images were reconstructed and analyzed with the instrument software-NRecon and CTAn (v1.10.1.0, Bruker, Kontich, Belgium). Quantitative measurements and 3D models of GS were performed by segmentation of drug particles in 8-bit greyscale images. The particles of GS with pores inside were treated as a whole body for analysis of dimensions of particles. The dimensions of particles were calculated as a diameter of the maximal sphere fitted within single particles. The quantitative analysis was performed on six volumes of interests for each type of fibers.

\subsection{Thermal Analysis}

Thermogravimetric analysis (TGA) was conducted on a Q5000 Analyzer (TA Instruments, New Castle, DE, USA). Samples of around $10 \mathrm{mg}$ were placed on a platinum pan and were then heated to $530{ }^{\circ} \mathrm{C}$, at a rate of $10^{\circ} \mathrm{C} / \mathrm{min}$ under a nitrogen flow of $25 \mathrm{~mL} / \mathrm{min}$.

Differential Scanning Calorimetry (DSC) was conducted on a Q2000 apparatus (TA Instruments, New Castle, DE, USA). Samples of around $5 \mathrm{mg}$ were sealed in an aluminum pan and heated in the 
range of $-80^{\circ} \mathrm{C}$ up to $80^{\circ} \mathrm{C}$, at a rate of $10^{\circ} \mathrm{C} / \mathrm{min}$ under a nitrogen flow of $50 \mathrm{~mL} / \mathrm{min}$. For GS samples, the range of temperature was from $20^{\circ} \mathrm{C}$ to $200{ }^{\circ} \mathrm{C}$, with a similar machine configuration. Three samples from the printed fibers were used for this analysis. Only one heating cycle was performed. The percentage of crystallinity of PCL was calculated based on the following formula:

$$
\text { Crystallinity }=\frac{H_{s}}{H_{f_{c}} * R} \cdot 100 \%
$$

where $H_{S}$ is the melting enthalpy of a sample, $R$ is the PCL content in a sample and $H_{f \mathcal{C}}$ is the melting enthalpy of $100 \%$ crystalline PCL which is $142 \mathrm{~J} / \mathrm{g}$ [25].

\subsection{Mechanical Testing}

Tensile and compression tests were performed on MicroTester 5943 (Instron, Norwood, MA, USA). For tensile testing, the $20 \mathrm{~mm}$-long fibers were used. The tensile rate was set to $0.1 \mathrm{~mm} / \mathrm{min}$ and the distance between grips was $10 \mathrm{~mm}$; according to guidelines about elastic modulus measurement in ISO527-1 the chosen tensile rate should provide 1\% of strain per min. For compression testing, samples with $4 \mathrm{~mm}$ height and $4 \mathrm{~mm}$ diameter were tested at a rate of $0.5 \mathrm{~mm} / \mathrm{min}$. Five samples were tested for each analysis per group. The elastic modulus (EM) was calculated from the stress-strain curve generated by the machine's software (BlueHill 3, v3.72, Instron, Norwood, MA, USA). A MATLAB code was written to calculate the slope of the best fit line between the $2.5 \%$ and $7.5 \%$ strain.

\subsection{Drug Loading and Release Test}

The drug loading test was performed for the first and last disks printed in the batch for the drug release experiment (20 samples in batch). The samples were dissolved in chloroform overnight. The solution was thoroughly mixed, and $1 \mathrm{~mL}$ aliquots were withdrawn and mixed with $5 \mathrm{~mL}$ of phosphate buffer solution (PBS), then stirred for an hour to assure the dissolution of GS in the PBS. The mixture was then left for 30 min without stirring until a clear phase separation was achieved. Finally, an aliquot from the PBS phase was removed and was used for measurements of the concentration of GS.

For the drug release test, three samples per group were sterilized by dipping in ethanol (96\%), then the samples were dried in the laminar air flow cabinet (BioWizard Silver SL-200 Blue Series Class II, KOJAIR@, Mänttä-Vilppula, Finland) for a couple of hours and placed in $4.5 \mathrm{~mL}$ of PBS at $37.5^{\circ} \mathrm{C}$. At each time point the buffer solution was replaced with a fresh one. The collected PBS, with the extracted (released) GS, was used in measurements of concentration of GS.

In both experiments (drug loading and drug release), the GS assay was performed by mixing collected PBS aliquots with phthaldialdehyde reagent and iso-propanol in a 1:1:1 (v/v) ratio [26-28]. This mixture was left for 45 min to react. A calibration curve was prepared and was used to measure the content of the GS in the aliquots using a UV-VIS spectrometer Nicolet Evolution 60 (Thermo Fisher Scientific, Madison, WI, USA) at a $332 \mathrm{~nm}$ wavelength. The range used for the calibration curve was between $294.8 \mathrm{up}$ to $2.3 \mathrm{ug} / \mathrm{mL}$ with $\mathrm{R}^{2}$ of 0.9984 . All measure aliquots were in the selected range of detection.

\subsection{Minimum Inhibitory Concentration (MIC)}

The minimum inhibitory concentration-the lowest concentration of the eluate that completely inhibited visible growth of methicillin-sensitive Staphylococcus (S.) aureus (ATCC 25923) after $24 \mathrm{~h}$ of incubation at $37^{\circ} \mathrm{C}$-was determined in 96-well microplates. The serial 1:1 dilutions of the eluates were made in TSB at a volume of $90 \mu \mathrm{L} /$ well in 96-well microtiter plates. Each well was then inoculated with $10 \mu \mathrm{L}$ of the bacterial inoculum. To prepare the inoculum, S. aureus (ATCC 25923) was cultured in $30 \mathrm{~g} / \mathrm{L}$ TSB under aerobic conditions at $37^{\circ} \mathrm{C}, 220 \mathrm{rpm}$ for $4 \mathrm{~h}$ to reach the exponential phase (around $10^{8} \mathrm{CFU} / \mathrm{mL}$ ). This was estimated by spectrophotometric turbidity measurements at $595 \mathrm{~nm}$ using a 
Thermo Scientific Multiskan Sky microplate spectrophotometer (Thermo Fisher Scientific, Waltham, MA, USA) and by CFU counts on TSA plates. The concentration was then adjusted to $10^{7} \mathrm{CFU} / \mathrm{mL}$. The effect was examined after incubation at $37^{\circ} \mathrm{C}$ at $200 \mathrm{rpm}$ for $24 \mathrm{~h}$ in a humidified incubator.

\subsection{Minimum Biofilm Inhibitory Concentration (MBIC)}

The anti-biofilm effects of the eluates were assessed prior to biofilm formation. This was carried out as described earlier by Fallarero et al. [29] with some modifications. One modification was that given the large number of samples, 348-well microplates were used instead of 96-well microplates [30]. The bacterial inoculum was prepared as described in Section 2.8, and adjusted to a concentration of $10^{6} \mathrm{CFU} / \mathrm{mL}$. To form biofilms, these exponentially grown cultures $\left(10^{6} \mathrm{CFU} / \mathrm{mL}\right)$ were added into flat-bottomed 348-well microplates (Nunclon $\Delta$ surface, Nunc, Roskilde, Denmark) simultaneously with the eluates at a 1:1 ratio, in a final volume of $40 \mu \mathrm{L}$. As a negative control, a PBS-TSB solution was used at a ratio 1:1, in a final volume of $40 \mu \mathrm{L}$. The biofilm inhibitory effect was examined after incubation at $37^{\circ} \mathrm{C}, 200 \mathrm{rpm}$ for $18 \mathrm{~h}$.

The effect on the biofilm viability was measured by following the protocol by Skogman et al. [31], using resazurin redox staining. Briefly, the planktonic suspension was removed and biofilms were washed twice with $50 \mu \mathrm{L}$ of PBS, then they were stained with $20 \mu \mathrm{M}$ resazurin for $20 \mathrm{~min}$ at $200 \mathrm{rpm}$ at room temperature, and the fluorescence was measured at $\Lambda_{\text {excitation }}=560 \mathrm{~nm}$ and $\Lambda_{\text {emission }}=590 \mathrm{~nm}$ (Varioskan LUX Multimode Microplate Reader, Thermo Scientific, Waltham, MA, USA).

\subsection{Adherence of Bacteria to the Surface}

The adherence of bacteria was evaluated by the static biofilm method with some modifications according to the protocol by Hiltunen et al. [32]. A sterile Whatman filter paper $(70 \mathrm{~mm}$ diameter, qualitative grade 2, GE Healthcare, Little Chalfont, U.K.) was placed on TSA plate $(90 \times 15 \mathrm{~mm})$. For biofilm formation, the pre-culture of methicillin-sensitive S. aureus (ATCC 25923) - $1 \mathrm{~mL}$ of colonies suspended into $100 \mathrm{~mL}$ of TSB - were grown under aerobic conditions at $37^{\circ} \mathrm{C}$ and $220 \mathrm{rpm}$ for $20 \mathrm{~h}$, and then diluted 1:10 in TSB to obtain approximately $10^{8} \mathrm{CFU} / \mathrm{mL}$ of bacteria. The filter paper was inoculated with $1.5 \mathrm{~mL}$ of the bacterial dilution. This was followed by the addition of the printed materials similar to the ones used for the drug release test, three samples corresponding to each mixing approach and each dosage of 2.5 and $7.5 \%$. As a negative control, PCL printed samples were used. This assembly was incubated under humidified aerobic conditions at $37^{\circ} \mathrm{C}$ for $24 \mathrm{~h}$. After incubation, samples were washed with TBS to remove any remaining planktonic cells and then they were transferred into falcon tubes containing $1 \mathrm{~mL}$ of $0.5 \%(w / v)$ Tween20:TSB solution. Subsequently, the tubes were sonicated in a water sonicator (Ultrasonic Cleaner 3800, Branson Ultrasonics, Danbury, CT, USA) at $25^{\circ} \mathrm{C}$, for $5 \mathrm{~min}$ at $35 \mathrm{kHz}$. The tubes were mixed vigorously for $20 \mathrm{~s}$ prior to and after the sonication step. Serial dilutions were performed from the resulting bacterial suspensions and plated on TSA (Neogen ${ }^{\circledR}$, Lansing, MI, USA) plates.

\subsection{Statistical Analyses}

Quantitative data were expressed as an average \pm standard deviation, number of samples are stated in each experiment. The statistical analyses were performed using the SPSS v.26 software and a one-way analysis of variance (ANOVA). TukeyHSD methods was used to measure statistical significance between groups, $p<0.05$ was considered to be statistically significant.

\section{Results and Discussion}

Recently, various research groups have been intensively working on producing drug loaded medical devices. Additionally, many methods have been used for drug incorporation into the carrier polymer. One of these methods is powder mixing of polymer and API [7]. As was mentioned before, the use of moderate particle sizes ranging from 250 to $600 \mu \mathrm{m}$ should provide a homogeneous distribution of the drug in the polymer matrix [22]. The GS powder used in the current study had 
a regular spherical shape with an average size below $100 \mu \mathrm{m}$. These spheres were hollow as some ruptured ones were observed (Figure 3A). The particles of PCL obtained after grinding of pellets had an irregular shape, as shown in Figure 3B. The used grinder crushes the polymer pellets by forcing them into a sieve with a $750 \mu \mathrm{m}$ diameter via centrifugal force. Thus, the produced particles should have a diameter smaller than $750 \mu \mathrm{m}$. This size was in the optimal range indicated by Saviano et al. [22].
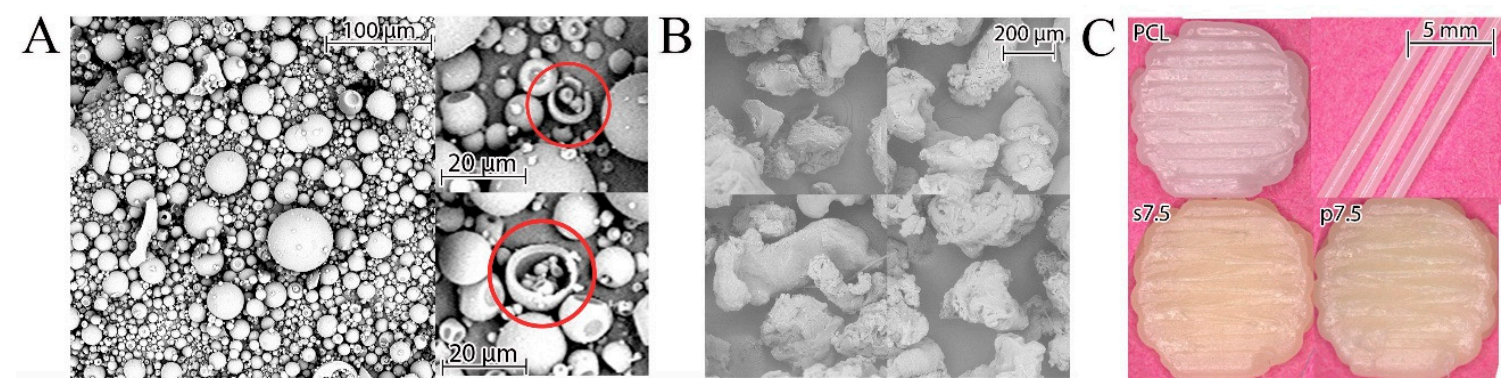

Figure 3. (A) SEM images of GS, red circles indicate some fractured spheres showing their hollow structure, (B) PCL particles obtained after grinding and (C) 3D printed fibers and disks.

A mixture of drug and polymer was used for preparing 3D printed samples with the theoretical drug concentration of $2.5 \%$ and $7.5 \%(w / w)$ (marked p2.5 and p7.5, respectively). Also, the samples printed from solvent cast materials with the same theoretical drug content (s2.5 and s7.5) were obtained. As a reference, samples made of pure PCL (pellets as received) were printed (Figure 3C).

The material was fed into the 3D printer and a preheating of $10 \mathrm{~min}$ at $100{ }^{\circ} \mathrm{C}$ was applied to assure the PCL melting. This time was also long enough to plasticize polymer-drug mixtures. PCL has a low melting point of $60{ }^{\circ} \mathrm{C}$ [33]. Thus, $100{ }^{\circ} \mathrm{C}$ was selected for the printing process, as it is higher than the melting point of the polymer and good flow characteristics through the nozzle were achieved.

The obtained fibers were measured by a digital caliper and had a diameter of $0.92 \pm 0.02 \mathrm{~mm}$. The beginning of the fiber was printed on part of the printing platform while the middle section was hung in the air to achieve a circular cross section as shown in Figure 2. Thus, the fibers were in slight tension which caused a slight decrease in their diameter compared to the inner diameter of the nozzle. Samples for compression testing, which were cut from 3D-printed structures with 100\% fill, had a diameter and height of $3.87 \pm 0.05 \mathrm{~mm}$ and $3.97 \pm 0.15 \mathrm{~mm}$, respectively. The drug loading and drug release samples had a diameter and height of $9.78 \pm 0.08 \mathrm{~mm}$ and $1.94 \pm 0.18 \mathrm{~mm}$, respectively. Their weight was around $135 \pm 10 \mathrm{mg}$.

\subsection{Thermal Properties}

The results of the TGA analysis are shown in Figure 4. GS itself shows content moisture as 15\% of weight loss, which occurred up to $130^{\circ} \mathrm{C}$. This was also reported by Rosenkrantz et al. [9]. After the water evaporation, the drug did not show any significant weight loss until $230^{\circ} \mathrm{C}$. At this temperature, a sudden decrease in weight was observed. These results demonstrated that the temperature of thermal degradation of GS is much higher than the processing temperature. The TGA results of pristine PCL showed that the polymer is thermally stable up to $400{ }^{\circ} \mathrm{C}$. A peak was observed at the drug degradation temperature (Figure 4C). This peak is proportional to the drug loading amount in the samples. Moreover, no differences were observed when comparing the drug mixing approaches. On the other hand, the pure PCL samples did not show any peak in this region. 

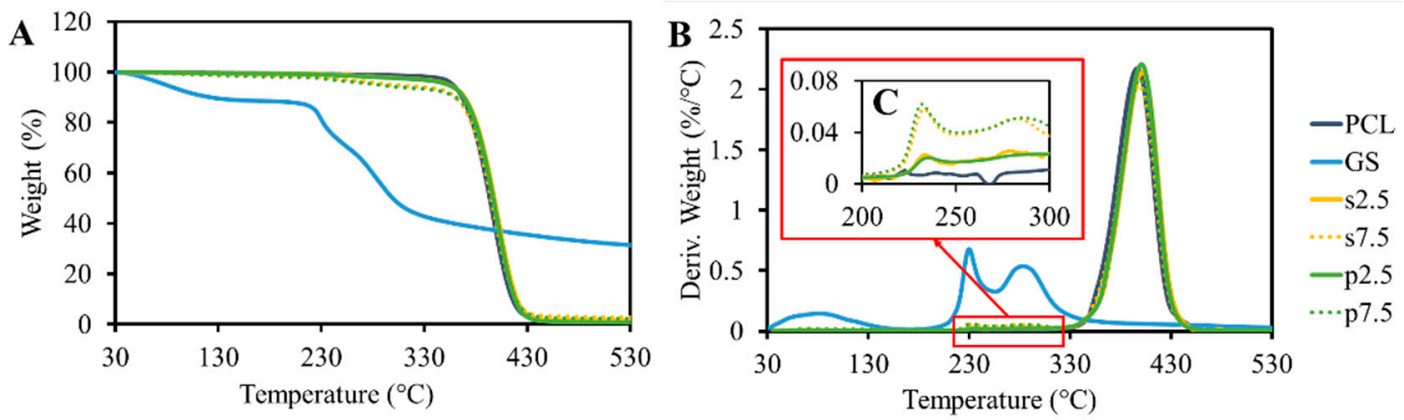

Figure 4. (A) TGA analysis of the produced PCL-GS samples, weight loss, (B) derivative weight loss, and (C) GS thermal degradation region.

DSC testing for the drug shows that there is no melting behavior between $0{ }^{\circ} \mathrm{C}$ and $200{ }^{\circ} \mathrm{C}$ for GS (Figure 5). However, a broad endothermic peak caused by the dehydration effect was present in the signal [9]. Moreover, DSC measurements showed that the loaded drug had no significant effect on the melting temperature (Figure 5 and Table 1 ). The crystallinity of the produced samples, which was calculated for the real polymer content, did not show any differences between the groups as no effect was caused by the presence of the drug particles (Table 1).

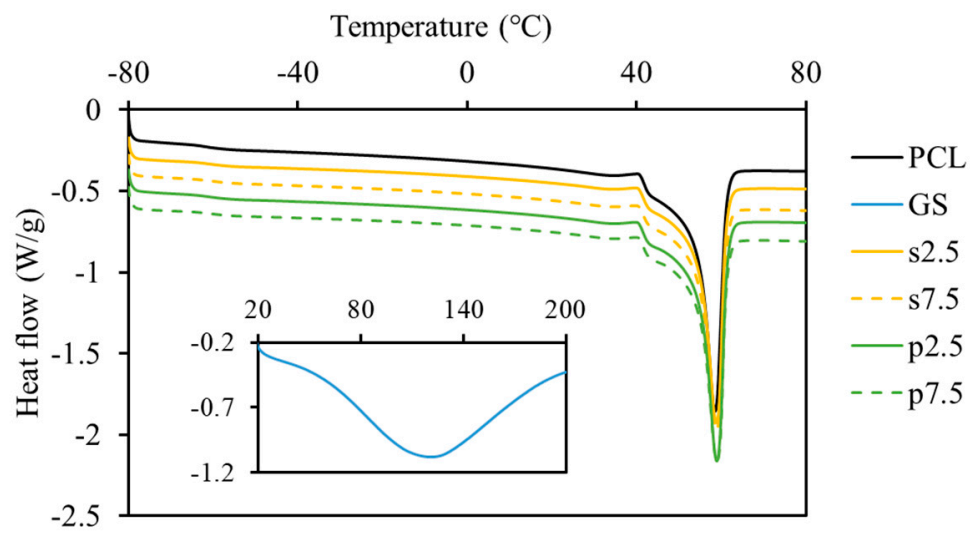

Figure 5. DSC analysis of produced PCL-GS samples (curves were shifted by $-0.1 \mathrm{~W} / \mathrm{g}$ ). The insert presents the curve for GS.

Table 1. DSC analyses results of produced PCL-GS samples.

\begin{tabular}{cccccc}
\hline & PCL & p2.5 & p7.5 & s2.5 & s7.5 \\
\hline $\mathbf{T}_{\mathbf{m}}\left({ }^{\circ} \mathbf{C}\right)$ & $58.8 \pm 0.3$ & $59.3 \pm 0.4$ & $59.1 \pm 0.1$ & $58.9 \pm 0.2$ & $59.1 \pm 0.2$ \\
Crystallinity (\%) & $41.5 \pm 0.2$ & $42.4 \pm 0.2$ & $41.4 \pm 1.2$ & $41.6 \pm 0.6$ & $42.9 \pm 1.1$ \\
\hline
\end{tabular}

\subsection{Mechanical Properties}

The mechanical properties of samples were tested using the tensile and compression test. The EM was used to compare groups. The single fibers were used for the tensile test. The pure PCL exhibited an EM of $119 \pm 3 \mathrm{MPa}$. For the drug loaded samples, it was $119 \pm 4,117 \pm 16,114 \pm 4$ and $111 \pm 3 \mathrm{MPa}$ for p2.5, p7.5, s2.5 and s7.5, respectively. As shown in Figure 6A, there was no statistically significant difference in the tensile EM between the pure PCL and drug loaded samples, nor between the two mixing approaches in the fibers. This suggests that the addition of the drug up to $7.5 \%(w / w)$ had no effect on the tensile mechanical properties of the produced fibers. Thus, such low concentrations had no effect on the integrity of the material. This corroborates with the results of the DSC test; no significant change in the crystallinity nor $\mathrm{T}_{\mathrm{m}}$ was observed suggesting a lack of changes in the microstructure of PCL matrix. 
A

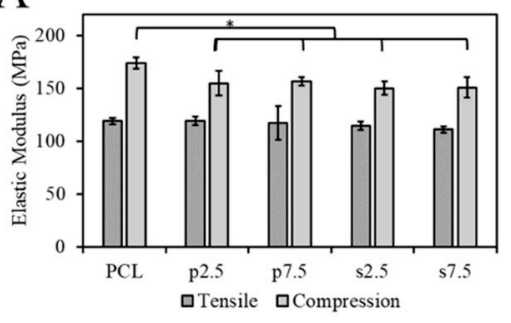

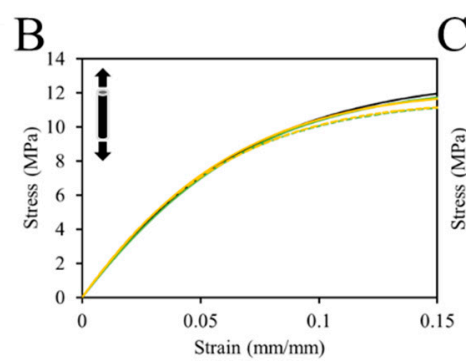

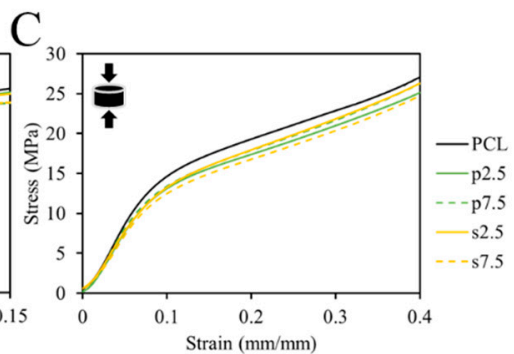

Figure 6. (A) Calculated elastic modulus of tensile and compression test $\left({ }^{*} p<0.05\right)$ and representative Stress-Strain curves for (B) tensile and (C) compression test for PCL and PCL loaded with GS samples.

On the other hand, 3D printed samples were used in the compression test. The compressive EM showed a statistically significant difference between the pure PCL and the drug loaded PCL in a slight decrease of c.a. $13 \pm 3 \%$ in the modulus in the 3D printed structures (Figure 6). However, no difference between the two mixing approaches was observed. The compressive EM of the pure PCL samples was $174 \pm 5 \mathrm{MPa}$. For the drug loaded samples, it was $155 \pm 12,157 \pm 4,150 \pm 6$ and $151 \pm 10 \mathrm{MPa}$ for p2.5, p7.5, s2.5 and s7.5, respectively. Similar results for 3D printed samples were reported by Tappa et al. [34] when loading polylactic acid (PLA) with GS. They loaded PLA with GS at $2.5 \%(w / w)$ and observed a decrease in the compression EM by $48 \%$.

\subsection{Drug Distribution}

The drug distribution within the polymeric matrix after 3D printing was evaluated by two techniques: SEM and $\mu \mathrm{CT}$. The dispersion of GS particles in the PCL varied when comparing the two mixing approaches. Figure 7 shows 3D reconstruction of the scanned fibers and SEM visualizations of the cross sections of fibers. The main difference between the two mixing approaches is the shape of the GS particles. It was observed that in the powder mixing approach, the GS shape was not altered during processing and the hollow spheres were visible in the $\mu \mathrm{CT}$ reconstruction (both $3 \mathrm{D}$ and cross section) (Figure 7A). On the other hand, the shape of the GS was altered and smaller solid particles were observed in the solvent casting approach (Figure 7A). This indicates that during the solvent casting approach the shell structure of GS was destroyed during the stirring process. Additionally, some agglomerates were visible in the powder mixing approach indicated by red circles in Figure 7A. Such agglomerates were not present in the solvent casting approach and the drug distribution was much more homogenous. 3D reconstructions of fibers printed from pristine PCL showed no pores in the produced fibers.

Quantitative data calculated from the $\mu \mathrm{CT}$ reconstructions are shown in Figure S1. As can be seen in Figure S1A, the GS particles' dimension shows similar trend in both the powder mixing and the solvent casting approaches regardless of the drug loading. However, when comparing the two approaches, the quantitative data shows differences in particles' dimensional distribution which confirms the visual observations shown in Figure 7A. Around $60 \%$ of the total volume of GS particles has a dimension below than $16 \mu \mathrm{m}$ and $22 \mu \mathrm{m}$ in the solvent casting and the powder mixing approaches, respectively (Figure S1B). Moreover, the average GS particles' dimension is $18.42 \pm 2.42,17.09 \pm 0.63$, $28.83 \pm 13.93$ and $21.09 \pm 5.70 \mu \mathrm{m}$ for s2.5, s7.5, p2.5 and p7.5, respectively (Figure S1C). It can be concluded that the solvent casting approach produced smaller drug particles with smaller standard deviation. On the contrary, the powder mixing approach produced larger drug particles (around 1.5 times) with larger standard deviation.

SEM images of cross sections of the single fibers shown in Figure 7B confirmed the results obtained by the $\mu \mathrm{CT}$ reconstruction in terms of drug distribution and particle size. However, in the SEM images the immiscibility of GS in the PCL matrix was much more apparent. This was found in all drug loaded groups. 

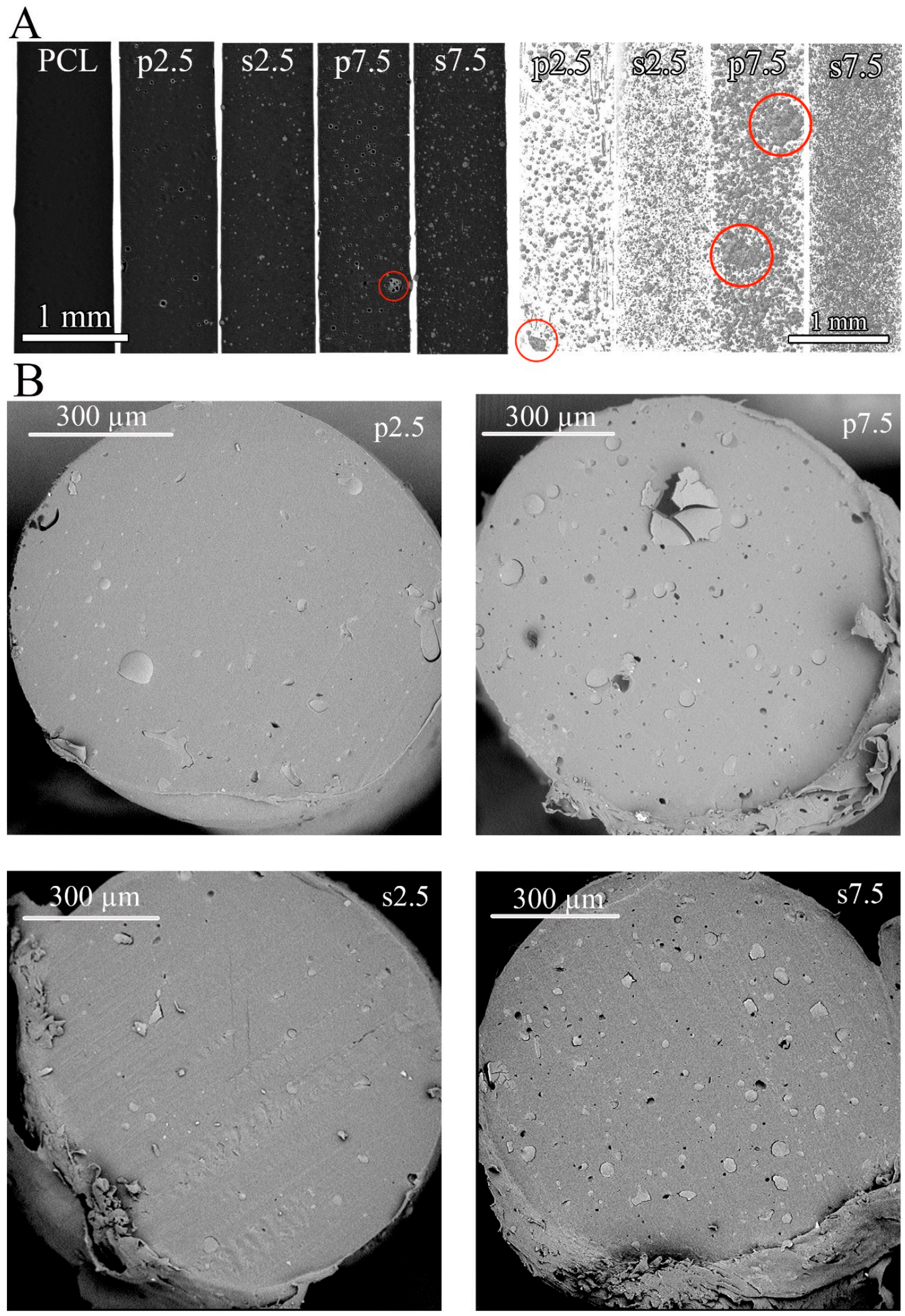

Figure 7. (A) Reconstructed cross sections of scanned fibers using $\mu \mathrm{CT}$ scanner (left) and 3D models showing only drug particles (right), red circles indicate agglomerates of GS within the PCL matrix and (B) SEM visualization of a cross sections prepared by cryosectioning from the printed fibers. 


\subsection{Drug Loading and Release Profile}

The drug loading efficiency is a very important parameter, especially from an economical point of view. The most desired are the technologies that provide a level close to $100 \%$. In our studies, around $100 \pm 11 \%$ of the GS was detected from the dissolved samples. The drug loading efficacy test showed that there was no statistically significant difference between the two mixing approaches in terms of drug loading efficiency as shown in Figure 8A. However, there was an observed difference in the release profile. As shown in Figure 8B, most of the loaded drug (around $90-100 \%$ of the loaded drug) was released from samples prepared by the solvent casting approach, while only $50-60 \%$ of the loaded drug was released from samples prepared by powder mixing after 62 days of release. This could be due to the difference in distribution of drug particles as illustrated in Figure 7. Moreover, the higher release can be explained due to the smaller drug particles. This contributed to the faster dissolution of the drug particles.
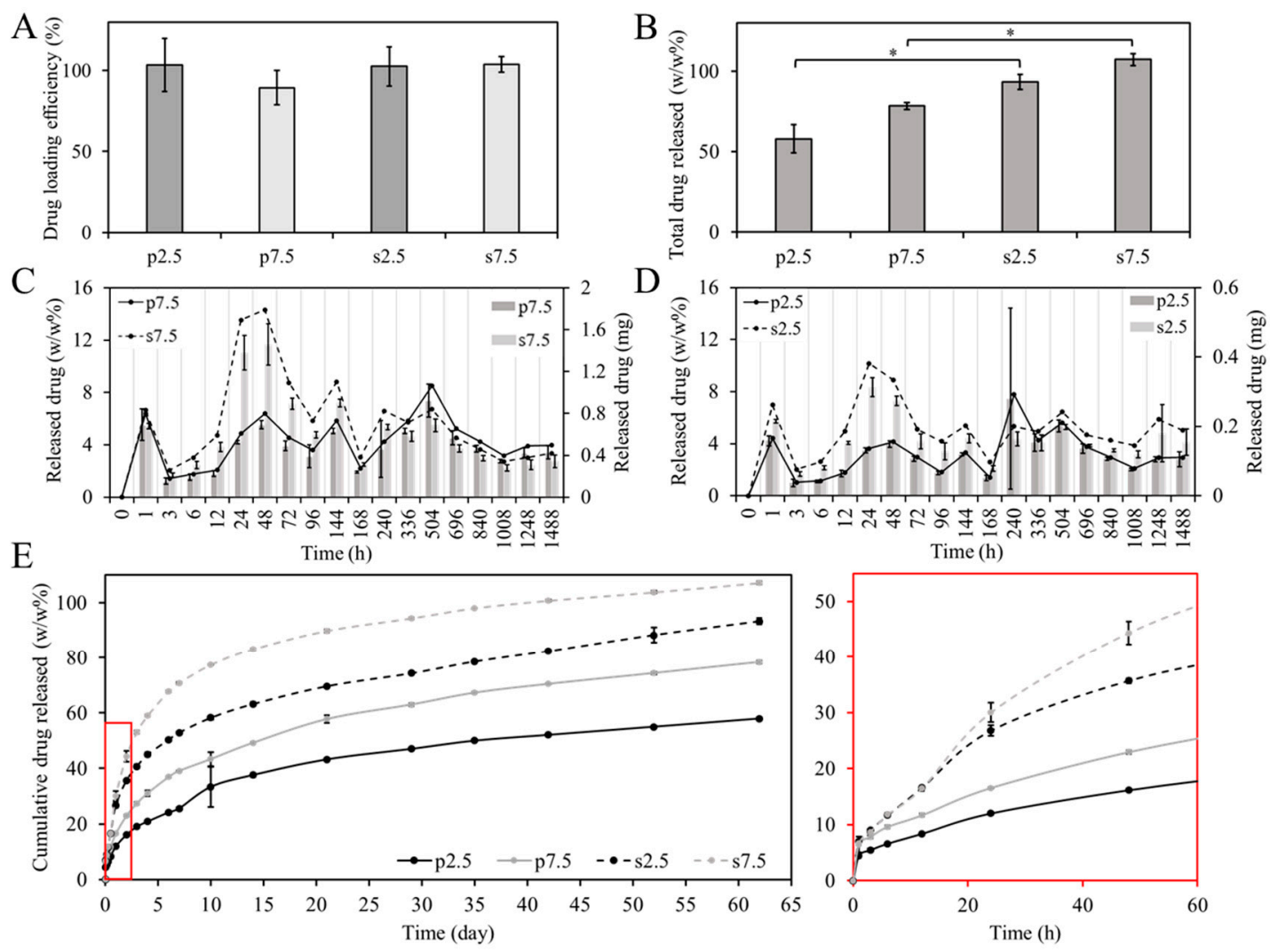

Figure 8. (A) Drug loading efficiency (ratio between the theoretical to the measured loading) in both mixing approaches; (B) Total amount of released drug expressed in the percentage of the $(w / w)$ ratio after 62 days $\left({ }^{*} p<0.05\right)$, released drug expressed in the percentage of the $(w / w)$ ratio (lines, left axis) and measured mass (bars, right axis) for samples with (C) $7.5 \%$, and (D) $2.5 \%$ of loaded drug and (E) Cumulative drug released $(w / w)$ from prepared samples for 62 days (for drug release test $\mathrm{n}=3$ ).

Moreover, Figure $8 \mathrm{C}, \mathrm{D}$ shows the weight percentage and the mass of the released drug at each time point. It can be noticed that most of the time points have a relatively similar release when comparing between the two mixing approaches, except for time points 24 and $48 \mathrm{~h}$. During this period, it is expected that the PBS would have diffused into the whole volume of the sample. At these two times points, a relatively higher release was observed from samples prepared by the solvent casting approach at the two drug loading concentrations. At $2.5 \%$ drug loading, the release was around two times higher in the solvent casting at the two time points. At $7.5 \%$ drug loading, the release was around three and two times higher in the solvent casting at $24 \mathrm{~h}$ and $48 \mathrm{~h}$, respectively. This caused 
the inflection in the drug release curve shown in Figure 8E. Furthermore, the shift in the drug release curve illustrated in Figure 8E between the two mixing approaches is almost the same between the two loading concentrations ( $2.5 \%$ and $7.5 \%)$. This demonstrates that the mixing approach affects the drug release profile. To better understand the release kinetics of each system, the release data were fitted into three commonly used models: (1) First order fitting, (2) Korsmeyer-Peppas model and (3) Higuchi model. The equations representing each model are presented in the supplementary material (Equations (S1)-(S3)). As can be seen from supplementary Figure S2 and Table S1, the Korsmeyer-Peppas model provides the best $R^{2}$ values. Thus, this model was selected to interpret the release kinetics. In this model the values of $\mathrm{n}$ represent the diffusion component which describe the drug release kinetics. For disk shaped samples, the $\mathrm{n}$ value for a Fickian diffusion mechanism should be located around $0.5[35,36]$. As can be seen in the supplementary Table S1, the $\mathrm{n}$ values obtained from the fitting were $0.42,0.52,0.39$ and 0.38 for s2.5, s7.5, p2.5 and p7.5, respectively. These values indicate a Fickian diffusion release kinematic.

\subsection{Antibacterial and Preventive Activity on Biofilm Formation}

As pointed out in the introduction, the potential application for these 3D printed materials would be the generation of delivery systems to prevent biofilm-related infections. This delivery systems are of special interest for prevention of bone infection as these avascular areas are unreachable for systemic antibiotics [37]. Because of this, the antibacterial and anti-biofilm properties of both the materials and eluates of the different time points was assessed. All eluates from all time points showed antibacterial activity against $S$. aureus (ATCC 25923). When comparing the two mixing methods, MIC values for the eluates confirmed the higher release from the samples corresponding to the solvent casting at the time points 24 and $48 \mathrm{~h}$ (Figure 8C,D). This was demonstrated by testing the antibacterial properties of the extracts after several dilutions, as shown in Table 2. At $2.5 \%(w / w)$, drug loading eluates showed two-fold higher antibacterial properties for samples prepared by solvent casting at both time points. On the other hand, at 7.5\% (w/w) the drug loading eluates showed 4- and 2-fold higher antibacterial properties at time points $24 \mathrm{~h}$ and $48 \mathrm{~h}$, respectively. This corroborates with the results obtained in the drug release measurements.

Table 2. Number of dilutions performed on eluates produced from the drug release test at time points $24 \mathrm{~h}$ and $48 \mathrm{~h}$ that still inhibit S. aureus (ATCC 25923) growth. Results corresponding to three biological replicates, with three technical replicates.

\begin{tabular}{ccccc}
\hline & s2.5 & p2.5 & s7.5 & p7.5 \\
\hline $\mathbf{2 4 ~ h}$ & $1: 4$ & $1: 2$ & $1: 16$ & $1: 4$ \\
$\mathbf{4 8 ~ h}$ & $1: 4$ & $1: 2$ & $1: 16$ & $1: 8$ \\
\hline
\end{tabular}

The effect of the eluates on the prevention of the biofilm formation as well as the anti-biofilm capability of the materials was also studied. It is essential to confirm the antimicrobial capability of both the eluates and the materials, to guarantee not only a prevention of biofilm formation onto the material but also in the surrounding tissue.

No differences were found in terms of prevention of biofilm formation between the two mixing approaches. Figure 9A shows the percentage of inhibition of the biofilm formation of the different time point eluates, calculated with respect to the biofilm formed by S. aureus (ATCC 25923) in the absence of eluate (bacterial control). All the eluates inhibited the biofilm formation for more than $90 \%$. This means that the concentration of GS in all the eluates is above the Minimum Biofilm Inhibitory Concentration (MBIC). This refers to the concentration needed for a compound to cause a $90 \%$ inhibitory effect on bacteria when a biofilm is developing [38]. 
A

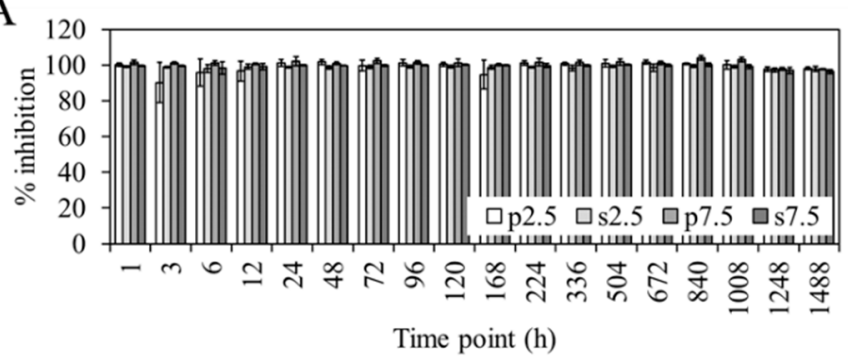

B

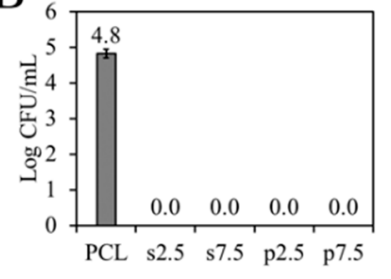

Figure 9. (A) Effect of the eluates on biofilm formation, percentage of inhibition calculated with respect to the bacterial control, (B) Number of viable attached S. aureus (ATCC 25923) (log CFU/mL) on PCL printed samples after $24-\mathrm{h}$ incubation. Results expressed as mean $\pm \mathrm{SD}$ of two biological replicates, with three technical replicates.

Figure 9B show the viable attached S. aureus (ATCC 25923) to the different materials. It can be seen how both approaches and both dosages completely prevent bacterial adherence when compared to the PCL control.

Our results are promising towards a success in the clinic, as both approaches provide a concentration of GS above the MBIC up to two months. Stravinskas, M et al. [39] studied if the in vitro release test of a synthetic bone graft loaded with GS predicted the performance of the material in vivo. In their study, the release of GS above the MIC for 28 days correlated with prevention of bone infection in the clinic. In our study, we have shown how both approaches guarantee a concentration of gentamicin not only above the MIC but also above the MBIC. However, it has to be taken into account that therapeutically, much higher concentrations are needed to reach bactericidal effect (four to eight times the MIC [40]). In that sense, the solvent casting would be a better approach to guarantee an efficient antimicrobial and anti-biofilm activity, as it has shown a significant higher release of the drug.

\section{Conclusions}

The production of drug loaded devices using 3D printing has shown promising results over the past few years. However, as most available 3D printers do not offer good mixing steps prior to printing, it is very important to prepare a homogenous mixture of the polymer and drug before loading it into the 3D printer. In this study, two commonly used approaches were compared, namely powder mixing and solvent casting. The material preparation steps varied among the two approaches. Powder mixing can be considered a more straight-forward process in case the polymer was supplied in a powder form. This is advantageous when moving towards industrialization. On the other hand, the solvent casting approach requires more steps. Additionally, the need for organic solvent can be considered a disadvantage in this process as using such solvent in industrial scale is not considered environmentally friendly. However, the solvent casting approach provided a more homogenous mixture with higher drug dispersing abilities compared to powder mixing. In this process the spherical shape of the drug particles was altered. Both mixing approaches resulted in similar thermal and mechanical properties. However, in the compression test the drug loaded samples in both approaches showed a decrease in the mechanical properties. The effect of drug loading on the mechanical properties of 3D printed constructs needs more investigation which can be considered in future work. Moreover, the drug release profile showed a significant difference. Samples prepared by solvent casting exhibited higher release characteristics. Despite both approaches produced efficient antibacterial and anti-biofilm materials, the solvent casting approach displayed a higher antimicrobial effect due to the higher release.

Supplementary Materials: The following are available online at http://www.mdpi.com/1996-1944/13/15/3364/s1, Figure S1. (a) Range frequency calculated as a percentage volume in a certain dimensional range, with (b) illustrating the cumulative frequency highlighting when percentage volume reaches around $60 \%$ of the total volume and (c) Particles' average dimension at different drug loading percentages, Equations S1-S3: illustrating models to describe the release kinetics, Table S1: Results from curve fitting the release curves to different models illustrating the value of each model parameters and the $R^{2}$ values. The model with the highest $R^{2}$ is 
highlighted, Figure S2. Release kinetics of GS from PCL samples according to (a) First order fitting, (b) fitting to Korsmeyer-Peppas model and (c) fitting to Higuchi model.

Author Contributions: Conceptualization B.S., I.R., Ż.G. and E.C., investigation, writing-review \& editing B.S., I.R., Ż.G., E.C, W.Ś., P.C. and A.F., Supervision B.V., K.B., E.C., W.Ś., P.C. and A.F., Funding acquisition B.V., K.B., W.Ś., P.C. and A.F. All authors have read and agreed to the published version of the manuscript.

Funding: This research was funded by the research project PRINT-AID, the EU Framework Program for Research and Innovation within Horizon 2020-Marie Sklodowska-Curie Innovative Training Networks under grant agreement No. 722467.

Conflicts of Interest: The authors declare no conflict of interest.

\section{References}

1. Crump, S.S. Apparatus and Method for Creating Three-Dimensional Objects. U.S. Patent 5,121,329A, 9 June 1992.

2. Araújo, M.R.P.; Sa-Barreto, L.L.; Gratieri, T.; Gelfuso, G.M.; Cunha-Filho, M. The Digital Pharmacies Era: How 3D Printing Technology Using Fused Deposition Modeling Can Become a Reality. Pharmaceutics 2019, 11, 128. [CrossRef]

3. Ilyés, K.; Kovács, N.K.; Balogh, A.; Borbás, E.; Farkas, B.; Casian, T.; Marosi, G.; Tomuță, I.; Nagy, Z.K. The applicability of pharmaceutical polymeric blends for the fused deposition modelling (FDM) 3D technique: Material considerations-printability-process modulation, with consecutive effects on in vitro release, stability and degradation. Eur. J. Pharm. Sci. 2019, 129, 110-123. [CrossRef] [PubMed]

4. Goyanes, A.; Allahham, N.; Trenfield, S.J.; Stoyanov, E.; Gaisford, S.; Basit, A.W. Direct powder extrusion 3D printing: Fabrication of drug products using a novel single-step process. Int. J. Pharm. 2019, 567, 118471. [CrossRef] [PubMed]

5. Shim, J.-H.; Kim, M.-J.; Park, J.Y.; Pati, R.G.; Yun, Y.-P.; Kim, S.E.; Song, H.-R.; Cho, D.-W. Three-dimensional

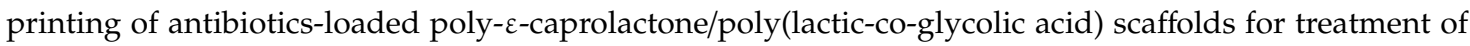
chronic osteomyelitis. Tissue Eng. Regen. Med. 2015, 12, 283-293. [CrossRef]

6. Fanous, M.; Gold, S.; Muller, S.; Hirsch, S.; Ogorka, J.; Imanidis, G. Simplification of fused deposition modeling 3D-printing paradigm: Feasibility of 1-step direct powder printing for immediate release dosage form production. Int. J. Pharm. 2020, 578, 119124. [CrossRef]

7. Shaqour, B.; Samaro, A.; Verleije, B.; Beyers, K.; Vervaet, C.; Cos, P. Production of Drug Delivery Systems Using Fused Filament Fabrication: A Systematic Review. Pharmaceutics 2020, 12, 517. [CrossRef]

8. Chang, H.-I.; Perrie, Y.; Coombes, A.G.A. Delivery of the antibiotic gentamicin sulphate from precipitation cast matrices of polycaprolactone. J. Control. Release 2006, 110, 414-421. [CrossRef]

9. Rosenkrantz, B.E.; Greco, J.R.; Hoogerheide, J.G.; Oden, E.M. Gentamicin Sulfate. In Analytical Profiles of Drug Substances; Florey, K., Ed.; Academic Press: New York, NY, USA, 1981; Volume 9, pp. 295-340.

10. Percival, S.L.; Suleman, L.; Vuotto, C.; Donelli, G. Healthcare-associated infections, medical devices and biofilms: Risk, tolerance and control. J. Med. Microbiol. 2015, 64, 323-334. [CrossRef]

11. Paharik, A.E.; Horswill, A.R. The Staphylococcal Biofilm: Adhesins, Regulation, and Host Response. In Virulence Mechanisms of Bacterial Pathogens; John Wiley \& Sons, Ltd.: Hoboken, NJ, USA, 2016; pp. 529-566. ISBN 978-1-68367-071-1.

12. Hall-Stoodley, L.; Costerton, J.W.; Stoodley, P. Bacterial biofilms: From the Natural environment to infectious diseases. Nat. Rev. Microbiol. 2004, 2, 95-108. [CrossRef]

13. Olson, M.E.; Ceri, H.; Morck, D.W.; Buret, A.G.; Read, R.R. Biofilm bacteria: Formation and comparative susceptibility to antibiotics. Can. J. Vet. Res. Rev. Can. Rech. Vet. 2002, 66, 86-92.

14. Kumar, A.; Alam, A.; Rani, M.; Ehtesham, N.Z.; Hasnain, S.E. Biofilms: Survival and defense strategy for pathogens. Int. J. Med Microbiol. 2017, 307, 481-489. [CrossRef]

15. Singh, S.; Singh, S.K.; Chowdhury, I.; Singh, R. Understanding the Mechanism of Bacterial Biofilms Resistance to Antimicrobial Agents. Open Microbiol. J. 2017, 11, 53-62. [CrossRef] [PubMed]

16. Arciola, C.R.; Campoccia, D.; Montanaro, L. Implant infections: Adhesion, biofilm formation and immune evasion. Nat. Rev. Microbiol. 2018, 16, 397-409. [CrossRef] [PubMed]

17. Weiser, J.R.; Saltzman, W.M. Controlled release for local delivery of drugs: Barriers and models. J. Control. Release 2014, 190, 664-673. [CrossRef] 
18. Rumiński, S.; Ostrowska, B.; Jaroszewicz, J.; Skirecki, T.; Włodarski, K.; Święszkowski, W.; Lewandowska-Szumiel, M. Three-dimensional printed polycaprolactone-based scaffolds provide an advantageous environment for osteogenic differentiation of human adipose-derived stem cells. J. Tissue Eng. Regen. Med. 2018, 12, e473-e485. [CrossRef] [PubMed]

19. Ostrowska, B.; Luca, A.D.; Moroni, L.; Swieszkowski, W. Influence of internal pore architecture on biological and mechanical properties of three-dimensional fiber deposited scaffolds for bone regeneration. J. Biomed. Mater. Res. Part A 2016, 104, 991-1001. [CrossRef]

20. Teo, E.Y.; Ong, S.-Y.; Khoon Chong, M.S.; Zhang, Z.; Lu, J.; Moochhala, S.; Ho, B.; Teoh, S.-H. Polycaprolactone-based fused deposition modeled mesh for delivery of antibacterial agents to infected wounds. Biomaterials 2011, 32, 279-287. [CrossRef]

21. Tariq, S.; Rahim, A.; Muhammad, N.; Rahman, S.U.; Azhar, U.; Sultana, K.; Sharif, F.; Siddiqi, S.A.; Zaman, M.; Rehman, F. Controllable delivery from gentamicin loaded polycaprolactone/grafted silica nanoparticles composite mats. J. Mol. Liq. 2019, 290, 111205. [CrossRef]

22. Saviano, M.; Aquino, R.P.; Del Gaudio, P.; Sansone, F.; Russo, P. Poly(vinyl alcohol) 3D printed tablets: The effect of polymer particle size on drug loading and process efficiency. Int. J. Pharm. 2019, 561, 1-8. [CrossRef]

23. Abedalwafa, M.; Wang, F.; Wang, L.; Li, C. Biodegradable Poly-Epsilon-Caprolactone (PCl) For Tissue Engineering Applications: A Review. Rev. Adv. Mater. Sci. 2013, 34, 123-140.

24. Górecka, Ż.; Idaszek, J.; Kołbuk, D.; Choińska, E.; Chlanda, A.; Święszkowski, W. The effect of diameter of fibre on formation of hydrogen bonds and mechanical properties of 3D-printed PCL. Mater. Sci. Eng. C 2020, 114, 111072. [CrossRef]

25. Diaz-Gomez, L.; Concheiro, A.; Alvarez-Lorenzo, C.; García-González, C.A. Growth factors delivery from hybrid PCL-starch scaffolds processed using supercritical fluid technology. Carbohydr. Polym. 2016, 142, 282-292. [CrossRef] [PubMed]

26. Virto, M.R.; Frutos, P.; Torrado, S.; Frutos, G. Gentamicin release from modified acrylic bone cements with lactose and hydroxypropylmethylcellulose. Biomaterials 2003, 24, 79-87. [CrossRef]

27. Sampath, S.S.; Robinson, D.H. Comparison of New and Existing Spectrophotometric Methods for the Analysis of Tobramycin and Other Aminoglycosides. J. Pharm. Sci. 1990, 79, 428-431. [CrossRef] [PubMed]

28. Mosselhy, D.A.; Ge, Y.; Gasik, M.; Nordström, K.; Natri, O.; Hannula, S.-P. Silica-Gentamicin Nanohybrids: Synthesis and Antimicrobial Action. Materials 2016, 9, 170. [CrossRef]

29. Fallarero, A.; Skogman, M.; Kujala, J.; Rajaratnam, M.; Moreira, V.M.; Yli-Kauhaluoma, J.; Vuorela, P. (+)-Dehydroabietic Acid, an Abietane-Type Diterpene, Inhibits Staphylococcus aureus Biofilms in Vitro. Int. J. Mol. Sci. 2013, 14, 12054-12072. [CrossRef]

30. Gilbert-Girard, S.; Savijoki, K.; Yli-Kauhaluoma, J.; Fallarero, A. Optimization of a High-Throughput 384-Well Plate-Based Screening Platform with Staphylococcus aureus ATCC 25923 and Pseudomonas aeruginosa ATCC 15442 Biofilms. Int. J. Mol. Sci. 2020, 21, 3034. [CrossRef]

31. Skogman, M.E.; Vuorela, P.M.; Fallarero, A. Combining biofilm matrix measurements with biomass and viability assays in susceptibility assessments of antimicrobials against Staphylococcus aureus biofilms. J. Antibiot. 2012, 65, 453-459. [CrossRef]

32. Hiltunen, A.K.; Vuorela, P.M.; Fallarero, A. Bisphosphonates offer protection against prosthetic joint infections caused by Staphylococcus aureus and Staphylococcus epidermidis biofilms. J. Drug Deliv. Sci. Technol. 2017, 40, 136-141. [CrossRef]

33. Abdelrazek, E.M.; Hezma, A.M.; El-khodary, A.; Elzayat, A.M. Spectroscopic studies and thermal properties of PCL/PMMA biopolymer blend. Egypt. J. Basic Appl. Sci. 2016, 3, 10-15. [CrossRef]

34. Tappa, K.; Jammalamadaka, U.; Weisman, J.A.; Ballard, D.H.; Wolford, D.D.; Pascual-Garrido, C.; Wolford, L.M.; Woodard, P.K.; Mills, D.K. 3D Printing Custom Bioactive and Absorbable Surgical Screws, Pins, and Bone Plates for Localized Drug Delivery. J. Funct. Biomater. 2019, 10, 17. [CrossRef]

35. Algahtani, M.S.; Mohammed, A.A.; Ahmad, J.; Saleh, E. Development of a 3D Printed Coating Shell to Control the Drug Release of Encapsulated Immediate-Release Tablets. Polymers 2020, 12, 1395. [CrossRef] [PubMed]

36. Ritger, P.L.; Peppas, N.A. A simple equation for description of solute release I. Fickian and non-fickian release from non-swellable devices in the form of slabs, spheres, cylinders or discs. J. Control. Release 1987, 5, $23-36$. [CrossRef] 
37. Butini, M.E.; Cabric, S.; Trampuz, A.; Di Luca, M. In vitro anti-biofilm activity of a biphasic gentamicin-loaded calcium sulfate/hydroxyapatite bone graft substitute. Colloids Surf. B Biointerfaces 2018, 161, 252-260. [CrossRef]

38. Gupta, A. Biofilm Quantification and Comparative Analysis of MIC (Minimum Inhibitory Concentration) \& MBIC (Minimum Biofilm Inhibitory Concentration) Value for Different Antibiotics against E. coli. Int. J. Curr. Microbiol. Appl. Sci. 2015, 4, 198-224.

39. Stravinskas, M.; Horstmann, P.; Ferguson, J.; Hettwer, W.; Nilsson, M.; Tarasevicius, S.; Petersen, M.M.; McNally, M.A.; Lidgren, L. Pharmacokinetics of gentamicin eluted from a regenerating bone graft substitute. Bone Jt. Res. 2016, 5, 427-435. [CrossRef] [PubMed]

40. Tam, V.H.; Kabbara, S.; Vo, G.; Schilling, A.N.; Coyle, E.A. Comparative Pharmacodynamics of Gentamicin against Staphylococcus aureus and Pseudomonas aeruginosa. Antimicrob. Agents Chemother. 2006, 50, 2626-2631. [CrossRef] [PubMed]

(C) 2020 by the authors. Licensee MDPI, Basel, Switzerland. This article is an open access article distributed under the terms and conditions of the Creative Commons Attribution (CC BY) license (http://creativecommons.org/licenses/by/4.0/). 